\title{
Neuroprotección del extracto hidroalcohólico de las hojas de Satureja brevicalyx 'wayra muña' en un modelo animal de hiperoxia e hipoxia-isquemia
} Satureja brevicalyx 'wayra muna' leaves hydroalcoholic extract neuroprotection in a hyperoxia and ischemia-hypoxia animal model

\author{
Rody Arias-Rodríguez¹, Jimmy Pascual Toma-Zárate1 , Enrique Javier Aguilar-Felices ${ }^{1}$, \\ Emilio Germán Ramírez-Roca ${ }^{1}$, Roberto Shimabuku-Azato ${ }^{2,3}$, Silvia Suárez-Cunza ${ }^{4}$ \\ Escuela de Formación Profesional de Farmacia y Bioquímica, Universidad Nacional San Cristóbal de Huamanga, Ayacucho, Perú. \\ ${ }^{2}$ Departamento Académico de Pediatría, Universidad Nacional Mayor de San Marcos, Lima. \\ ${ }^{3}$ Instituto Nacional de Salud del Niño, Lima, Perú. \\ ${ }^{4}$ Centro de Investigación de Bioquímica y Nutrición, Facultad de Medicina, Universidad Nacional Mayor de San Marcos, Lima, Perú.
}

\begin{abstract}
Resumen
Objetivos: Evaluar el efecto neuroprotector del extracto hidroalcohólico de hojas de Satureja brevicalyx 'wayra muña' en ratas sometidas a hiperoxia, y su progenie sometidas a hipoxia isquemia. Diseño: Experimental. Lugar: Área de Cirugía Experimental, Instituto Nacional de Salud del Niño, y Laboratorios del Centro de Investigación de Bioquímica y Nutrición, Facultad de Medicina, Universidad Nacional Mayor de San Marcos, Lima, Perú. Material biológico: Rattus novegicus de la cepa Holtzman, hojas secas de Satureja brevicalyx 'wayra muña'. Intervenciones: Tratamiento con el extracto en dos modelos: hiperoxia en ratas hembras adultas, e hipoxia en la progenie de ratas madre tratadas. Se realiza ANOVA y prueba de Tukey. Principales medidas de resultados: Actividad de superóxido dismutasa (SOD), niveles de glutatión (GSH) total y niveles de sustancias reactivas al ácido tiobarbitúrico (TBARS). Resultados: En las ratas tratadas con el extracto se observó disminución significativa de TBARS y participación de GSH y SOD. Conclusiones: Nuestros resultados sugieren que el extracto hidroalcólico de Satureja brevicalyx ejerce efecto neuroprotector en condición de hiperoxia e hipoxia experimental, mediante la mitigación de la lipoperoxidación como parámetro de daño oxidativo, con participación del GSH y la actividad de SOD como mecanismos de defensa antioxidante.

Palabras clave: Agentes neuroprotectores, estrés oxidativo, superóxido dismutasa, glutatión, hiperoxia, anoxia, satureja.

\section{Abstract}

Objectives: To determine Satureja brevicalyx 'wayra muna' leaves hydroalcoholic extract neuroprotective effect in rats subjected to hyperoxia and its progeny to hypoxia ischemia. Design: Experimental. Setting: Department of Experimental Surgery, National Institute of Child Health, and Biochemistry and Nutrition Research Center Laboratory, Faculty of Medicine, Universidad Nacional Mayor de San Marcos, Lima, Peru. Biological material: Rattus novegicus Holtzman strain, Satureja brevicalyx 'wayra muna' dry leaves. Interventions: Treatment with the extract had two models: hyperoxia in adult female rats and hypoxia in the offspring of rats treated. Both ANOVA and Tukey test were performed. Main outcome measures: Superoxide dismutase (SOD) activity, total glutathione (GSH) and thiobarbituric acid reactive substances (TBARS) levels. Results: Rats treated with the extract showed significant decrease in TBARS and participation of GSH and SOD. Conclusions: Our results suggest that Satureja brevicalyx hydroalcoholic extract exerts neuroprotective effect in hyperoxia and hypoxia conditions by alleviating experimental lipid peroxidation, an oxidative damage parameter, with participation of GSH and SOD activity as antioxidant defense mechanisms.
\end{abstract}

Key words: Neuroprotective agents, oxidative stress, superoxide dismutase, glutathione, hyperoxia, anoxia, satureja.

An Fac med. 2012;73(3):215-9

\section{INTRODUCCIÓN}

La alta incidencia de disfunción neurológica asociada a diversos eventos clínicos y el reconocimiento de la importancia de productos naturales que coadyuvan en las estrategias terapéuticas han motivado diversos estudios para identificar y demostrar los efectos neuroprotectores de la variada riqueza de nuestros recursos naturales.
El término 'neuroprotección' se utiliza para referirse a cualquier medida profiláctica que se inicia durante el período peri-isquémico, con el fin de mejorar la supervivencia neuronal ${ }^{(1)}$. Entre los principales mecanismos bioquímicos que intervienen en este proceso se incluyen los flujos iónicos transmembrana y el aumento de calcio intracelular, excitotoxicidad, la formación de radicales libres, la producción de peroxinitrito, la liberación de mediadores de la inflamación, la disfunción mitocondrial, entre otros ${ }^{(1)}$. Condiciones ambientales adversas, como hipoxia, isquemia, hiperoxia, temperatura elevada, compuestos tóxicos, estrés oxidativo, pueden ser iniciadores de este proceso ${ }^{(2)}$.

El oxígeno, molécula indispensable para mantener la vida de los organismos aerobios, puede potencialmente 
ser el factor iniciador de un proceso denominado estrés oxidativo que puede llevar a la muerte; esta es la paradoja del oxígeno. Esta molécula da lugar a la formación de especies reactivas del oxígeno (EROs), tanto en condiciones de hipoxia como de hiperoxia. Hoy se sabe que las EROs están implicadas en la patogenia de muchas enfermedades, siendo capaces de dañar, en forma reversible e irreversible, diversas biomoléculas muy importantes para la estructura y función de las células -lípidos, proteínas y los ácidos nucleicos-, lo que se traduce a nivel del sistema nervioso en muerte neuronal (3). Esto ocurre al producirse un desbalance en los mecanismos reguladores y homeostáticos, o al comprometer la integridad estructural de la célula.

La literatura muestra evidencias importantes sobre el papel potencial de las EROs en patologías neurológicas agudas como la isquemia ${ }^{(4)}$, y enfermedades crónicas neurodegenerativas como el Alzheimer, Parkinson y esclerosis lateral amiotrófica ${ }^{(4-6)}$. El recién nacido es especialmente vulnerable a los efectos tóxicos del oxígeno. La adaptación a la vida extrauterina implica un cambio de una $\mathrm{PaO} 2$ de 25 a $43 \mathrm{mmHg}$ durante la vida intrauterina ${ }^{(7)}$ a otra que se duplica, 60 a $80 \mathrm{mmHg}{ }^{(8)}$.

La hipoxia isquemia en el periodo perinatal sigue siendo una de las causas más prevalentes de secuelas neurológicas en niños ${ }^{(9)}$. El recién nacido posee un cerebro en desarrollo, lo que determina una mayor sensibilidad neuronal a los neurotransmisores, un metabolismo férrico acentuado en las células gliales, lo que junto a su carencia relativa de antioxidantes les hace particularmente susceptibles al efecto de los radicales libres ${ }^{(9)}$. El cerebro neonatal es un órgano con alta tasa metabólica y de extracción de oxígeno, lo que le hace más rápidamente vulnerable a la hipoxia-isquemia ${ }^{(7,8)}$.

Una alternativa válida para la prevención de estos desequilibrios causados por los EROs son los recursos vegetales, por la variedad de metabolitos secundarios oxigenados, nitrogenados y azufrados con capacidad de atrapar EROs. Estos comprenden carotenoides, compuestos nitrogenados (alcaloides, aminas) e incluso terpenoides, entre otros; pero, quizás los más reconocidos por su actividad antioxidante son los de naturaleza fenólica: ácidos fenólicos, flavonoides, quinonas, cumarinas y taninos ${ }^{(10)}$.

Entre las innumerables especies vegetales que producimos de forma silvestre con ancestral aplicación medicinal es la Satureja brevicalyx Epl 'wayra muña'; los usos populares se deben a sus efectos analgésico, antiinflamatorio, antimicrobiano y para afecciones gastrointestinales. Las sustancias químicas identificadas que caracterizan su actividad biológica son principalmente sustancias de naturaleza fenólica ${ }^{(11)}$; participan en los procesos que forman parte de los mecanismos antioxidantes, lo que podría contribuir a disminuir el desbalance oxidativo y de esa manera disminuir el daño neurológico que se presenta en las diversas situaciones de estrés ${ }^{(12)}$.

En el presente estudio, se ha evaluado la actividad neuroprotectora del extracto hidroalcohólico de las hojas de Satureja brevicalyx Epl 'wayra muña' en el cerebro de ratas, para lo cual se planteó dos modelos experimentales: una de hiperoxia ( $100 \%$ de oxígeno) como agente causante del daño neurológico, y un segundo modelo de hipoxia isquemia en la progenie procedente de ratas hembras tratadas con el extracto de la wayra muña procedente del departamento de Ayacucho.

\section{MÉTODOS}

Las plantas de Satureja brevicalyx Epl 'wayra muña' provinieron del centro poblado de Ccallpapata, Distrito de Huamanguilla, Provincia de Huanta, Departamento de Ayacucho, ubicado a 3800 m.s.n.m., recolectados durante los meses de febrero a marzo de 2007. Previo secado de las hojas en un lugar con adecuada ventilación y bajo som- bra, se procedió a la selección de las hojas perfectamente secas para ser molidas y luego maceradas por un periodo de 7 días. Se pesó $3 \mathrm{~kg}$ y se maceró en 9 litros de alcohol al 80\%. Se filtró con un sistema de vacío utilizando papel Whatman, se concentró en baño maría y finalmente se secó en una estufa a $40^{\circ} \mathrm{C}$.

Para el modelo animal, se empleó ratas albinas hembras Rattus novegicus de la cepa Holtzman, de pesos entre 200 y 250 g, procedentes del Instituto Nacional de Salud. Las ratas fueron previamente acondicionadas al ambiente por 10 días. Los animales fueron mantenidos en jaulas individuales, en un ambiente controlado de temperatura, humedad ciclo de luz-oscuridad 12 horas, recibiendo una dieta balanceada obtenida del mismo Instituto Nacional de Salud de Lima. Estas condiciones fueron aplicadas en ambos modelos experimentales.

Para el primer modelo, se empleó 36 ratas separadas aleatoriamente en dos grupos experimentales de 18 ratas cada grupo. El grupo 1 recibió agua y el grupo 2, el extracto hidroalcohólico de la hojas Satureja brevicalyx Epl 'wayra muña' disuelto en agua a una concentración de $3 \mathrm{mg} / \mathrm{mL}$. El consumo de agua y el extracto fue ad líbitum por un periodo de 30 días. Después de este periodo, cada grupo fue dividido en dos subgrupos:

- 1a - Los que consumieron agua (control).

- $1 b$ - Los que consumieron agua y fueron sometidos a hiperoxia.

- 2a - Los que consumieron extracto.

- $2 \mathrm{~b}$ - Los que consumieron extracto y fueron sometidos a hiperoxia.

La injuria neurológica consistió en someter a las ratas de los grupos $1 \mathrm{~b}$ y $2 \mathrm{~b}$ en una incubadora hermética, con suministro de oxígeno al 100\% durante 8 horas. Después de 24 horas, todos los animales fueron sacrificados por decapitación; se extrajo los cerebros. Se preparó el homogenizado de cerebro a una concentración de $10 \%$ en buffer fosfato 
$0,05 \mathrm{M}$ y pH 7,4, en un homogenizador tipo Potter Elvjhem con émbolo de teflón, a una temperatura de 4 ㅇ $\mathrm{C}$; se centrifugó a $2000 \mathrm{rpm}$, durante 5 minutos, y el sobrenadante fue utilizado para los ensayos bioquímicos.

Para el segundo modelo, se formó dos grupos de 6 ratas Holtzman hembras de peso homogéneo 200 a $250 \mathrm{~g}$, primerizas: El grupo I (GI) fue tratado con agua corriente y el grupo II (GII), con extracto de wayra muña $3 \mathrm{mg} / \mathrm{mL}$.

El tratamiento se inició 10 días previos a la gestación y se continuó durante la etapa de la gestación; en ambos casos se administró el líquido ad líbitum. Los recién nacidos (12 por grupo) fueron divididos aleatoriamente en cuatro grupos, dos de ellos sometidos a hipoxiaisquemia (H-I), según se explica.

- Dos formados a partir del grupo GI: CI (sin H-I) y CIII (con H-I)

- Dos formados a partir del grupo GII: CII ( $\sin \mathrm{H}-\mathrm{I})$ y CIV (con $\mathrm{H}-\mathrm{I})$.

El modelo de hipoxia-isquemia consistió en ligar la arteria carótida izquierda seguida de la exposición de hipoxia a $8 \%$ de $\mathrm{O} 2$ por 2 horas, a ratas de 7 días, con un rango de peso de 11 a $15 \mathrm{~g}{ }^{(13)}$. Luego de 24 h se les sacrificó por decapitación a todos los grupos. Se recogió los cerebros y se siguió el mismo procedimiento antes descrito.

Se midió los niveles de glutatión mediante el método químico con DTBN (ácido 5,5'-ditiobis (2-nitrobenzoico)), según Beutler AF y Ellman GL, con ligeras modificaciones ${ }^{(14)}$. Se midió 950 $\mu \mathrm{L}$ de homogenizado y se le añadió 50 $\mu \mathrm{L}$ de ácido tricloroacético (ATC) al $100 \%$; se agitó y se llevó a centrifugación. Se separó $200 \mu \mathrm{L}$ de sobrenadante, que fue tratado con $1 \mathrm{~mL}$ de una mezcla de ácido glioxílico y ácido ascórbico; se incubó a $67^{\circ} \mathrm{C}$ por cinco minutos, se enfrió y se agregó $200 \mu \mathrm{L}$ de DTBN. Se leyó a $412 \mathrm{~nm}$.

La determinación de sustancias reactivas al ácido tiobarbitúrico (TBARS) se realizó siguiendo la técnica de Buege y Aust ${ }^{(15)}$. Se midió $0,3 \mathrm{~mL}$ de homogenizado, se precipitó las proteínas con

Tabla 1. Niveles de MDA, GSH y actividad de SOD como indicadores de la actividad neuroprotectora del extracto hidroalcohólico de las hojas de Satureja brevicalyx Epl. 'wayra muña' en un modelo de hiperoxia al $100 \%$ con ratas hembras $(n=9)$.

\begin{tabular}{|c|c|c|c|}
\hline Grupo & 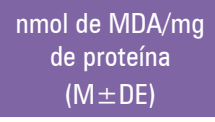 & $\begin{array}{c}\mathrm{nmol} \text { de } \mathrm{GSH} / \mathrm{mg} \\
\text { de proteína } \\
(\mathrm{M} \pm \mathrm{DE})\end{array}$ & $\begin{array}{l}\text { U SOD/ mg } \\
\text { de proteína } \\
(\mathrm{M} \pm \mathrm{DE})\end{array}$ \\
\hline $\begin{array}{c}\text { 1a } \\
\text { Agua }\end{array}$ & $0,835 \pm 0,033^{B}$ & $8,241 \pm 0,219^{c}$ & $14,52 \pm 0,29$ в \\
\hline $\begin{array}{c}1 \mathrm{~b} \\
\text { Agua }+ \text { hiperoxia }\end{array}$ & $1,006 \pm 0,051^{c}$ & $7,240 \pm 0,542^{A}$ & $13,38 \pm 0,37^{A}$ \\
\hline $\begin{array}{c}2 a \\
\text { Extracto }\end{array}$ & $0,628 \pm 0,078^{A}$ & $8,664 \pm 0,096^{D}$ & $15,13 \pm 0,36^{c}$ \\
\hline $\begin{array}{c}2 \mathrm{~b} \\
\text { Extracto }+ \text { hiperoxia }\end{array}$ & $0,852 \pm 0,096^{B}$ & $7,729 \pm 0,285^{\mathrm{B}}$ & $14,39 \pm 0,22^{B}$ \\
\hline
\end{tabular}

Datos expresados como la media \pm desviación estándar. Letras iguales por columnas: no hay significancia.

0,6 mL de ATC al 20\%, se colocó en baño maría hirviente por 15 minutos, se dejó enfriar y se agregó $0,9 \mathrm{~mL}$ de TBA al 0,67\% en $\mathrm{HCl} 0,25 \mathrm{~N}$; se llevó por segunda vez al baño maría hirviente, se enfrió en agua helada y se centrifugó a $6000 \mathrm{rpm}$ durante 10 minutos. Se llevó a lectura a $535 \mathrm{~nm}$. La actividad de superóxido dismutasa (SOD) fue medida con el método de Marklund y Marklund ${ }^{(16)}$, basado en la capacidad de inhibir la autoxidación del pirogalol que se produce en medio alcalino con formación del radical superóxido. En la cubeta de $1 \mathrm{~mL}$ se colocó $950 \mu \mathrm{L}$ de buffer tris $\mathrm{HCl} 0,05 \mathrm{M}$, a pH 8,2, se incubó por un minuto a temperatura de $37^{\circ} \mathrm{C}$ y se añadió pirogalol $2 \mathrm{mM}$, produciéndose un cambio de absorbancia de $0,02+/-10 \%$ por minuto, leído en el espectrofotómetro a una longitud de onda $420 \mathrm{~nm}$. Para la lectura de las muestras, se colocó una cantidad de homogenizado que disminuyera el cambio de absorbancia a la mitad, también $+/-10 \%$. Una unidad SOD es igual al $50 \%$ del cambio de absorbancia del pirogalol.

La determinación de proteínas se hizo mediante el método de Lowry ${ }^{(17)}$.

Para el análisis estadístico, todos los datos obtenidos fueron expresados como la media \pm desviación estándar y sometida al análisis de varianza (ANOVA) y prueba de Tukey, mediante el programa SPSS versión 15.0 en entorno Windows. Se consideró un $\mathrm{p}<0,05$.

\section{RESULTADOS}

Del primer modelo, en la tabla 1 se observa un incremento significativo $(\mathrm{p}<0,05)$ de TBARS en el tratamiento de hiperoxia al $100 \%$ y una recuperación significativa $(p<0,05)$ por el tratamiento con el extracto con niveles semejantes al grupo control 1a. El mismo comportamiento se observa en el resultado de los niveles de GSH. Sin embargo, en este caso la recuperación fue significativamente menor al grupo control 1a. En la misma tabla se observa que la injuria hiperóxica también provocó disminución significativa de la actividad de SOD, actividad que fue recuperada bajo el tratamiento con el extracto, expresando una actividad semejante al grupo control. Es interesante notar que el grupo 2a exhibió una variación significativa en los tres parámetros medidos con respecto a los demás grupos de tratamiento.

Los resultados del segundo modelo (tabla 2) muestran una tendencia a la disminución de las TBARS en los grupos tratados con el extracto de wayra 
Tabla 2. Niveles de MDA, GSH y actividad de SOD como indicadores de la actividad neuroprotectora del extracto hidroalcohólico de las hojas de Satureja brevicalyx Epl. 'wayra muña' en un modelo de hipoxia-isquemia en ratas de 7 días de nacidos $(n=12)$.

\begin{tabular}{cccccc} 
& \multicolumn{2}{c}{ Sin tratamiento H - I } & & \multicolumn{2}{c}{ Con tratamiento H - I } \\
\cline { 2 - 3 } \cline { 6 - 6 } & C I (agua) & C II (extracto) & & C III (agua) & C IV (extracto) \\
TBARS (nmol/g tej) & $95,7+12,0$ & $77,5+22^{2}$ & & $102,4+17^{a}$ & $84,4+17,0$ \\
GSH ( $\mu \mathrm{g} / \mathrm{g}$ tej) & $398,2+61$ & $346,5+112$ & & $322,6+113$ & $354,5+44$ \\
SOD (U SOD/g tej) & $492,3+38,0$ & $521,5+93,0$ & & $472,5+71$ & $515,0+106$ \\
\hline
\end{tabular}

$\mathrm{a}=$ con significancia estadística.

muña, alcanzando significancia entre los grupos CII y CIII. El efecto del extracto a la hipoxia sobre la actividad de SOD no mostró variaciones significativas, pero una tendencia a un ligero incremento en los grupos experimentales. El mismo comportamiento se observó en los niveles de GSH total.

\section{DISCUSIÓN}

La neuroprotección está ligada a la posibilidad de intervención terapéutica dirigida, no a mejorar los síntomas de un déficit neurológico o cognitivo establecido, sino a prevenir su aparición, deteniendo las cascadas de eventos celulares y moleculares que desencadenan la muerte neuronal ${ }^{(18)}$. El estudio experimental desarrollado justamente sirve para evaluar este papel potencial de la Satureja brevicalyx.

Una situación de hiperoxia al 100\% lleva a un incremento de EROs, produciendo desbalance entre las reacciones prooxidantes frente a los sistemas antioxidantes enzimáticos y no enzimáticos. Considerando que el cerebro es un órgano rico en lípidos complejos especialmente insaturados, un parámetro importante debe ser la lipoperoxidación medida como TBARS. Entre los variados mecanismos antioxidantes, se puede medir enzimas y metabolitos.

El modelo experimental de hiperoxia en ratas adultas produjo un daño significativo $(\mathrm{p}<0,05)$, con incremento de lipoperoxidación (TBARS) que disminuyó significativamente por el tratamiento con el extracto hidroalcohólico de las hojas de Satureja brevicalyx. El papel neuroprotector del extracto también se observa cuando se administra sin inducción de daño oxidativo, lo que nos induce a considerarlo protector del proceso de lipoperoxidación en el cerebro, ya sea en un situación de estrés por hiperoxia o en condiciones normales.

En este mismo modelo de estrés oxidativo, el grupo control de hiperoxia (grupo 1b) mostró una significativa depleción $(\mathrm{p}<0,05)$ con respecto al grupo control (1a). El daño producido en el grupo con extracto e hiperoxia (grupo 1d) también mostró una disminución significativa respecto al grupo 1c. Sin embargo, este nivel fue significativamente mayor al grupo injuriado (grupo 1b), lo que refleja que sí existió un papel protector por la administración del extracto de wayra muña en situación de estrés oxidativo. Al igual que el indicador de TBARS, también se observó mayor contenido $(\mathrm{p}<0,05)$ de GSH total solo con la administración del extracto (grupo 1c). Luego, el extacto hidroalcohólico de las hojas de la Satureja brevicalyx coadyuva en el sistema de defensa antioxidante manteniendo los niveles de GSH elevados y evitando su descenso en situaciones de estrés.

En el caso de la actividad de la SOD, el daño por estrés oxidativo se observó con una reducción significativa $(\mathrm{p}<0,05)$ de la actividad. Otra vez, la respuesta al estrés en presencia del extracto mostró recuperación significativa comparable a la actividad del grupo 1 a. Es reiterativo el papel neuroprotector del extracto de Satureja brevicalyx aún en condición no injuriante.
La hipoxia puede generar EROs que directa o indirectamente estimulan factores inducibles por la hipoxia importantes para el desarrollo del cerebro ${ }^{(19)}$. Los resultados de la injuria por hipoxia-isquemia en la progenie de ratas tratadas con extracto de Satureja brevicalyx (tabla 2) dan cuenta que el extracto influyó favorablemente y de manera significativa en la neuroprotección, teniendo como indicador el TBARS, interpretándose como la capacidad de sus metabolitos secundarios de atravesar la barrera placentaria. Los resultados de glutatión total y actividad de SOD mostraron también una importante tendencia favorable para el efecto deseado, aunque sin significancia estadística, lo que puede interpretarse que su participación como mecanismo de defensa se realizó en vía de evitar un mayor daño peroxidativo a nivel lipídico. Estudios realizados en ratas recién nacidas han demostrado que el estrés oxidativo regula la capacidad antioxidante, especialmente en la expresión de enzimas antioxidantes ${ }^{(20)}$. Luego, debería considerarse que, si bien el metabolismo de defensa oxidativa del recién nacido es inmaduro y vulnerable a las EROs ${ }^{(21)}$, también es sabido que este metabolismo tiene una capacidad de adaptación y regulación, mostrando mejor respuesta inmediata que en el adulto, razón por la que sus mecanismos de defensa han actuado mitigando el grado de peroxidación lipídica.

Los metabolitos secundarios extraídos por la mezcla hidroalcohólica tiene componentes que incluye sustancias químicas de naturaleza fenólica y son las que poseen actividades biológicas ${ }^{(11)}$, las cuales participan en los procesos que forman parte de los mecanismos antioxidantes; de manera que este contenido puede contribuir a disminuir el desbalance oxidativo y de esa manera atenuar el daño neurológico que se presenta en las diversas situaciones de estrés ${ }^{(12)}$. Estudios previos en Satureja obovata var. obovata mostraron flavonoides con actividad vasodilatadora en la aorta de rata ${ }^{(22)}$. 
Nuestros resultados han demostrado el potencial neuroprotector del extracto hidroalcohólico de la Satureja brevicalyx 'wayra muña' proveniente del departamento de Ayacucho, tanto en situación de hiperoxia como en hipoxia. Las perspectivas de su aplicación y manejo dependerán de mayores estudios a nivel bioquímico y farmacológico.

\section{AGRADECIMIENTOS}

Los autores agradecen al personal médico del Instituto Nacional de Salud del Niño, especialmente al Dr. Carlos Delgado y al personal que trabaja en el área de investigación del INSN.

\section{REFERENCIAS BIBLIOGRÁFICAS}

1. Hans P, Bonhomme V. Neuroprotection with anaesthetic agents. Curr Op Anaesthesiol. 2001;14(5):491-6.

2. Llanes J, Cancio M, Solis M, Rodriguez F, Llorente M, Rivas W. Variaciones de los leucocitos polimorfonucleares durante los periodos de isquemia y reperfusión de la cirugia coronaria. Rev Latinoam Tecnol Extracorp. 2002;2(2).

3. Gruber J, Schaffer S, Halliwell B. The mitochondrial free radical theory of ageing-where do we stand? Front Biosci. 2008;13:6554-79.

4. Martínez-Lazcano JC, Boll-Woehrlen MC, Hernández-Melesio $M$, Rubio-Osornio $M$, SánchezMendoza MA, Ríos C, Pérez-Severiano F. Estrés oxidativo en las enfermedades neurodegenerativas. En: Álvarez Fernández G, Bustos Jaimes I, Castañeda Patlán C, Guevara Fonseca J, Romero Álvarez I, Vázquez Meza H. (eds). Mensaje Bioquímico. 2010;34:43-59.

5. Henchcliffe C, Flint Beal M. Mitochondrial biology and oxidative stress in Parkinson disease pathogenesis. Nature Clin Pract Neurol. 2008;4(11):600-9.

6. Vargas MR, Johnson DA, Sirkis DW, Messing A Johnson JA. Nrf2 activation in astrocytes protects against neurodegeneration in mouse models of familial amyotrophic lateral sclerosis. J Neurosc. 2008;28(50):13574-81.

7. Phylactos AC, Ghebremeskel K, Costeloe AA, Leaf AA, Harbige LS, Crawford MA. Polyunsaturated fatty acids and antioxidants in early development. Possible prevention of oxygen-induced disorders. Eur J Clin Nutr. 1994;48(Suppl. 2):S17-S23.

8. Volpe JJ. Brain injury in the premature infant. Neuropathology, clinical aspects, pathogenesis, and prevention. Clin Perinatol. 1997;24:567-87.

9. Gidday JM., Fitzgibbons JC, Shah AR. Neuroprotection from ischemic brain injury by hypoxic preconditioning in the neonatal rat. Neurosci Lett. 1994;1768:221-4.

10. Bandonien D, Murkovicub M. The detection of radical scavenging compounds in crude extract of borage (Borago officinalis L.) by using an on-line HPLC- DPPH method. J Biochem Biophy Methods. 2002;53(1-3):65-70.

11. Soto M. Tamizaje fitoquímico y efecto analgésico de las hojas de la Satureja brevicalyx "Wayra muña". 1999. Tesis (QF) - UNSCH. Fac. Cs. Bs. Ayacucho - Perú.

12. Palomino R. Efecto antioxidante del extracto acuoso liofilizado de las hojas de la Satureja brevicalyx Epl "wayra muña". 2005. Tesis (QF) - UNSCH. Ayacucho-Perú.

13. Ota A, Ikeda T, Ikenoue T, Toshimori K. Sequence of neuronal responses assessed by immunohistochemistry in the newborn rat brain after hypoxiaischemia. Am J Obstet Gynecol. 1997;177:519-26.
14. Buege JA, Aust SD. Microsomal lipid peroxidation. Methods Enzimol. 1978;52:302-10.

15. BoyneAF, Ellman GL. Assay for glutation glutathione. Analyt Biochem. 1972;46:639-53.

16. Marklund S, Marklund G. Involvement of the superoxide anion radical in the autoxidation of pirogallol and a convenient assay for superoxide dismutase. Eur J Biochem. 1974;47:469-74.

17. Lowry OH, Rosebrough NJ, Farr AL, Randall RJ. Protein measurement with the folin phenol reagent. J Biol Chem. 1951;193:265-75.

18. Sirén L, Ehrenreich H. EPO - a novel concept for neuroprotection. Eur Arch Psychiatry Clin Neurosci. 2001;251:179-84.

19. Chávez OM, Flores ME, Chacón M, Chávez RJ. La neuroprotección en disfunción neurológica aguda. Nuevos enfoques terapéuticos dentro del campo de la inmunología del sistema nervioso central. Medicrit. 2005;2(8):179-85.

20. Richard RL, Auten L, Davis JM. Oxygen Toxicity and reactive oxygen species: the devil is in the details. Ped Res. 2009;66(2):121-7.

21. Ikonomidou C, Kaindl AM. Neuronal death and oxidative stress in the developing brain. antioxidants \& redox signaling. 2011;14(8):1535-50.

22. Sánchez de Rojas VR, Somoza B, Ortega T, Villar AM. Isolation of vasodilatory active flavonoids from the traditional remedy Satureja obovata. Planta Med. 1996;62(3):272-4

\section{Correspondencia:}

\section{Silvia Suárez Cunza}

Centro de Investigación de Bioquímica y Nutrición, Facultad de Medicina, Universidad Nacional Mayor de San Marcos, Lima, Perú

Av. Grau 755, Lima 1, Perú

Correo electrónico: ssuarezc@fmedicina.unmsm.pe 